\title{
INITIATION OF SECONDARY EXPLOSIVES MEASURED USING EMBEDDED ELECTROMAGNETIC GAUGES
}

\author{
C. Stennett, G. A. Cooper, P. J. Hazell, and G. Appleby-Thomas \\ Cranfield Defence and Security, Cranfield University, Shrivenham, SN6 8LA, UK
}

\begin{abstract}
There is considerable evidence that secondary explosive materials having a relatively large (10-12\%) proportion of HTPB binder do not exhibit DDT under cook-off. However, the understanding of the mechanisms controlling the growth of reaction in such experiments is incomplete. Most importantly, it is not known whether a mechanistic reason exists to preclude DDT; it is possible that existing techniques to explore cook-off simply do not offer the correct conditions to allow DDT to occur. We present experiments in which impacts were made against a RDX/HTPB PBX using a single-stage light gas gun. Electromagnetic particle velocity gauges were embedded within the targets at different distances from the impact face to record the onset of reaction, and in some cases detonation. These experiments were also performed against RDX/TNT targets. The time-resolved particle velocity histories have allowed comparison of some of the factors governing growth of reaction, and have provided run-to-detonation distance data for different impact stresses.
\end{abstract}

Keywords: Ignition and growth, PBX, particle velocity gauge, shock initiation.

PACS: 07.07.Df, 06.06-Ei, 47.40-Nm, 82.40-Fp.

\section{INTRODUCTION}

The particle velocity technique using embedded gauges has been extensively used over the past decades to explore the initiation and subsequent growth of reaction in energetic materials. In this technique conductors, embedded in a material that is subjected to shock loading, will acquire the local particle velocity. This can be measured by arranging a steady magnetic field so that a current is induced in the moving conductors. Early work [1-3] focused on the measurement of steady detonations, while more recently [4-7] interest has shifted to the initiation of explosives by gas-gun driven flyers. More recent work $[9,10]$ has developed this technique using the $50 \mathrm{~mm}$ singlestage gas gun at Shrivenham [8], and has included some characterization of the type of RDX/HTPB PBX that is currently of interest in the UK.
In this work we have refined our technique further in terms of target preparation and generation of the magnetic field, we have made improvements to measurement accuracy, and we have eased the experimental load in carrying out this type of experiment. Firings have been made in which RDX/TNT and RDX/HTPB charges were initiated, and the run-to-detonation distances have been measured. There appears to be superficially good agreement between our data and that for Composition B-3, but there remain unexplained details in the particle velocity histories we have recorded that demand further study.

\section{EXPERIMENTAL PROCEDURE}

The particle velocity gauges were of the Vorthman type [4], and followed the design 
described in [9], having seven Lagrangian elements varying in width from $13 \mathrm{~mm}$ to $7 \mathrm{~mm}$, and a single 'shock tracker'. The gauge arrays were etched from a flexible laminate of copper and polyimide, $87 \mu \mathrm{m}$ thick. The variation in length of the Lagrangian elements was no more than $0.3 \%$.

The gauges were encapsulated with two sheets of Mylar, bonded to the gauge using a lowviscosity epoxy adhesive to avoid premature loss of signals by the ionization from the incipient detonation. In early experiments, the encapsulating material was $50 \mu \mathrm{m}$ thick, yielding a gauge package thickness measured to be $185 \mu \mathrm{m}$. In later experiments the encapsulating material was $25 \mu \mathrm{m}$ thick, yielding a gauge package thickness of $140 \mu \mathrm{m}$.

The targets were all manufactured in two separate slabs, with one face angled to allow the gauge to be cemented between them at an angle to the impact face. The angled arrangement meant that an unobstructed portion of the target was presented to each gauge element along the impact axis. For these experiments the gauge angle was chosen to be $60^{\circ}$ to give a large depth over which the target was instrumented.

The RDX/TNT target components were cast from a single batch into a preheated mould that accurately defined the impact face and gauge mounting faces. The sample densities were measured using the Archimedes method, the RDX/TNT targets being $1.66 \pm 0.02 \mathrm{~g} / \mathrm{cc}$. and the PBX $1.58 \pm 0.06 \mathrm{~g} / \mathrm{cc}$. The PBX targets were cut from a single block, and lapped by hand to final dimensions. Additional characterization data are found in [11]. The angle of the gauge plane was measured with a vernier gauge to be $60 \pm 0.25^{\circ}$ for all parts.

The gauges were assembled onto one half of the target using an alignment jig and microscope, to an angular tolerance of $\pm 2 \mathrm{mrad}$ with respect to the impact face. A low viscosity epoxy adhesive was used to join the two target components together. Each target was covered by a $1 \mathrm{~mm}$ thick PMMA plate to aid mounting into the gun. The mounting alignment for this gun is typically $1.25 \mathrm{mrad}$.

The magnetic field was generated using a pair of electromagnets, energized at the moment of the experiment by switching a high-current power supply. In early experiments, two coils in the classical air-spaced Helmholtz arrangement were used, as described in [9]. While the results were good, several practical drawbacks were found: (a) the coils were destroyed on every experiment and new ones had to be made each time; and (b) to obtain a useful flux density in the target zone, the coils were driven with $\sim 10 \mathrm{~kW}$ power, leading to rapid Joule heating, and hence making calibration and testing of the coils difficult.

The later experiments were made using a pair of coils embedded in a steel and aluminium housing. The electromagnet geometry was designed using a commercial finite element code, in an iterative process that aimed to achieve high field strength and low supply power. The final design generated a field measured to be $110 \pm 2.0 \mathrm{mT}$ in a zone $20 \times 20 \times 20 \mathrm{~mm}$, centred on the axis of the gun. This was achieved using a $24 \mathrm{~V}$ DC battery bank, delivering 60A. A thermocouple embedded in one of the coils measured a rate increase of temperature of $0.2^{\circ} \mathrm{Cs}^{-1}$, and a measured rate of decrease in field strength of $0.1 \mathrm{mTs}^{-1}$. A flux meter was used to map the magnetic flux density, corroborated with measurement of the coil current using a clamp meter.

The eight sensing elements in the gauge package were connected by separate RG174 coaxial cables to two 4-channel oscilloscopes of $1 \mathrm{Ghz}$ bandwidth, sampling at $1.6 \mathrm{~ns}$ per point. The interconnecting cables were chosen to be impedance matched throughout. At a particle velocity of $1 \mathrm{kms}^{-1}$, the longest Lagrangian element, (13mm wide) would be expected to give a signal of $\sim 1.5 \mathrm{~V}$.

\section{RESULTS AND DISCUSSION}

It can be seen in Fig. 1 that the particle velocity on successive gauge elements increases, indicating the onset of reaction in the specimen.

The gross form of the record is consistent with expectation, but there are details that require further explanation. In particular, the particle velocity on each gauge overshoots after the initial rise, and this prevents analysis of the material flow immediately behind the shock. The rise times for the initial particle velocity excursion are consistent with an impact alignment of $2.5 \mathrm{mrad}$. In this 
experiment, the run to detonation occurs close to the last Lagrangian element at $13.2 \mathrm{~mm}$ from the impact face.

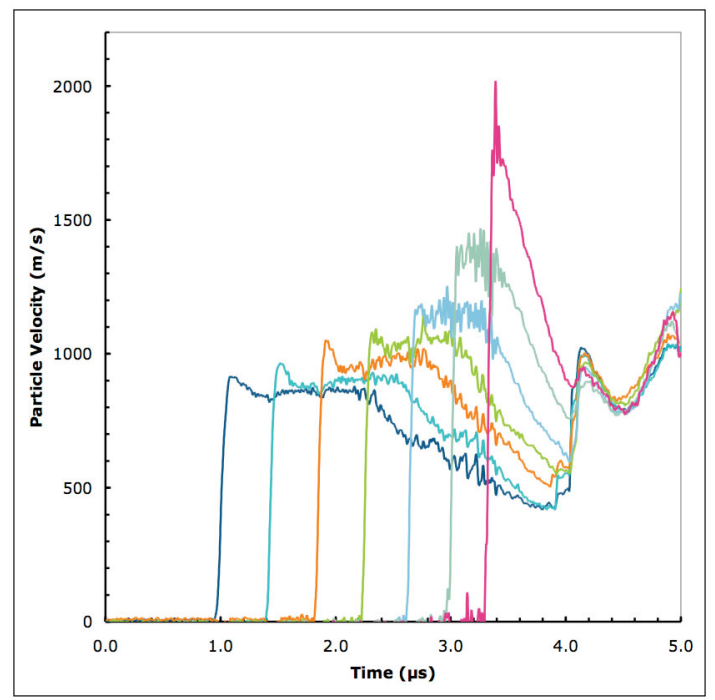

Figure 1. A typical particle velocity history for the PBX target. $\sigma=5.1 \pm 0.25 \mathrm{GPa}$. Gauge elements at 2.8, 4.5, $6.3,8.0,9.7,11.5,13.2 \mathrm{~mm}$ from the impact face. Detonation occurs between the gauges at 11.5 and $13.2 \mathrm{~mm}$.

The shock velocity can be obtained by linear fitting either to the shock tracker data (Fig. 2) or to the arrival times at the Lagrangian elements. The measured particle velocity, at the plateau after the initial rise and overshoot, can then be used to plot the unreacted Hugoniot for the specimen, in $U_{s}-U_{p}$ space as shown in Fig. 3.

The linear fits to the shock tracker arrival times were used to obtain run distances to detonation for each experiment as noted in Fig. 2. The known Hugoniots $[10,11]$ were used to obtain impact stress given the (linear) shock velocity prior to breakout of detonation. These yielded the plot shown in Fig. 4 for both compositions.

It can be seen from the plot in Fig. 4 that the RDX/TNT in this study has similar initiation properties to Composition B-3, over the range of impact stresses tested. The slight decrease in sensitivity compared with Composition B-3 is attributed to the use of HMX-free RDX in our material.

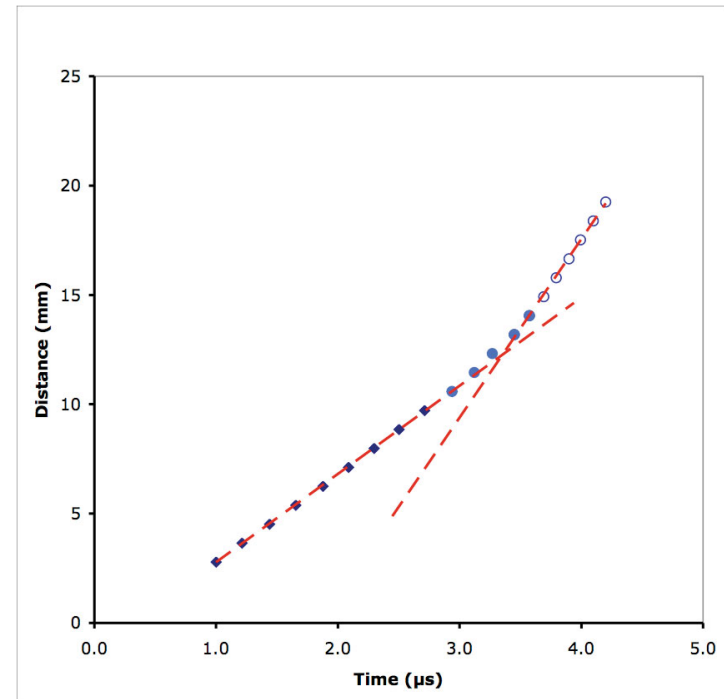

Figure 2. Shock tracker data for the shot shown in Figure 2. The linear fits give $U_{s}=4.03 \pm 0.1 \mathrm{~mm} \mu \mathrm{s}^{-1}, \mathrm{D}=$ $8.16 \pm 0.15 \mathrm{~mm} \mu \mathrm{s}^{-1}$. The transition between the two linear portions appears to be gradual. The crossing point for the linear fits suggests breakout of detonation at $12.4 \mathrm{~mm}$.

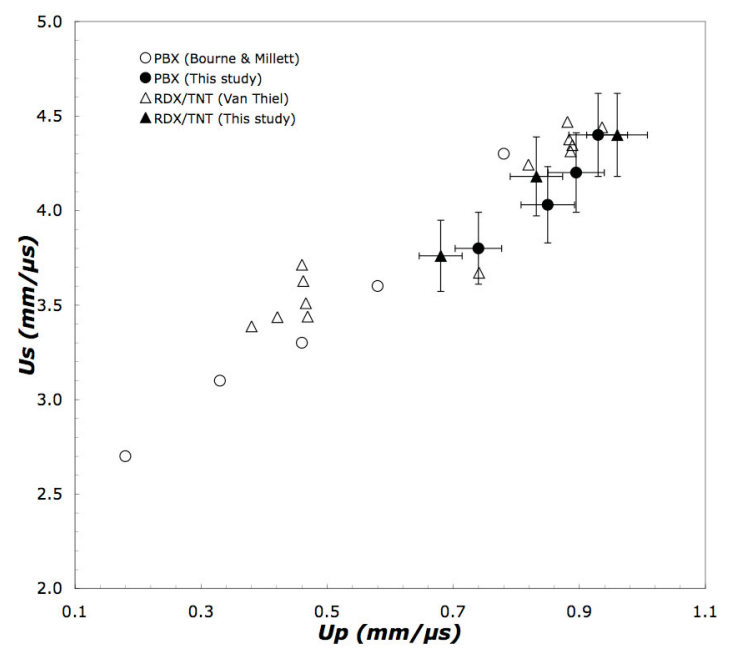

Figure 3. Hugoniot in $U_{s}-U_{p}$ space for PBX (circles) and RDX/TNT (triangles). Literature data (open symbols) shown for comparison. $U_{p}$ taken from the Lagrangian element closest to the impact face; $U_{s}$ derived from the shock arrival X-T plots, e.g. Figure 3.

The PBX composition is less sensitive than $\mathrm{RDX} / \mathrm{TNT}$, and a linear fit through the PBX data on the Pop-plot has a shallower slope compared to 
RDX/TNT. Hence, for low-stress impacts the run distance is longer. Extrapolation of the trend for the PBX upwards implies that at $\sim 7 \mathrm{GPa}$ the run distance to detonation for the PBX and for $\mathrm{RDX} / \mathrm{TNT}$ will be the same. It should be noted, however, that there are presently few data points in this study and confirmation of this trend must await further experiments.

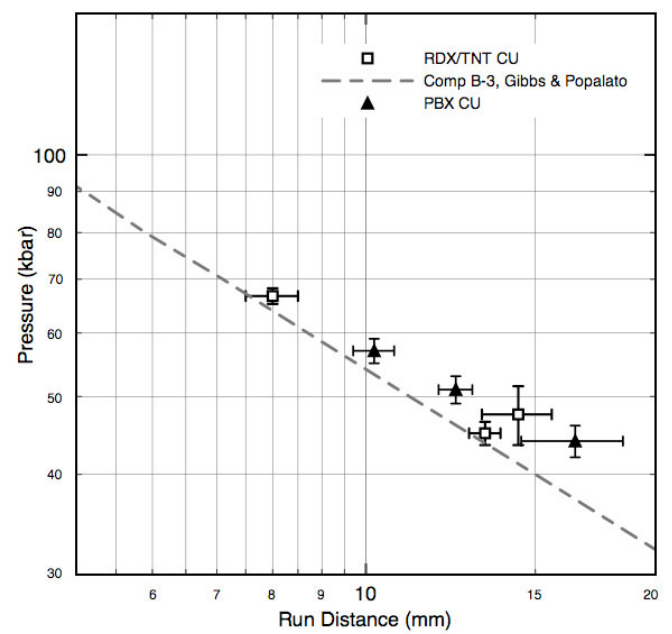

Figure 4. Pop-plot of impact pressure vs. run distance to detonation for all experiments. Literature data (Van Thiel) for Comp B-3 are given for comparison.

\section{CONCLUSIONS}

The embedded electromagnetic gauge technique has been implemented with reasonable success. The gross features of the initiation of the two secondary explosives have been captured, and there is good correspondence between literature data and the data from this study in both $U_{s}-U_{p}$ Hugoniot measurement and 'Pop-plot' measurement.

There are unexplained details in many of the experimental records, which obscure important features during the particle velocity history. In particular, the overshoots noted in Fig. 1 are apparent to some extent on all records. These are attributed to the shock impedance mismatch between the gauge package and the test specimen: a detailed study, following Cau [12], is proposed to examine these features in greater depth.

\section{ACKNOWLEDGEMENTS}

The authors would like to thank Prof. Neil Bourne, Miss Sally Gaulter, Dr William Proud and Dr Ron Hollands for their valuable advice.

\section{REFERENCES}

1. Zaitsev, V. M., Pokhil, P. F., and Shvedov, K. K., DAN SSSR, 132 (1960) p1339.

2. Dremin, A.N., and Shvedov, K.K., J. Appl. Mech. Tech. Phys., 2 (1964) p154.

3. Jacobs, S., and Edwards, D.J., "Experimental Study of the Electromagnetic Velocity-Gage Technique", Proceedings of the 5th Symposium (International) on Detonation, ONR Report No. ACR-184 (1970) p413.

4. Vorthman, J.E., Andrews G., and Wackerle, J., "Reaction Rates from Electromagnetic Gauge Data", in Proceedings of the 8th Symposium (International) on Detonation, ONR Report No. ACR-184 (1989) p413.

5. Cowperthwaite, M., and Rosenberg, J.T., "Lagrange Gage Studies of Detonation in some Intermolecular EA Based Explosives", in Proceedings of the 8th Symposium (International) on Detonation, ONR Report No. ACR-184 (1989) p111.

6. Leiper, G.A., Kirby, I.J., and Hackett, A., "Determination of Reaction Rates in Intermolecular Explosives using the Electromagnetic Particle Velocity Gauge", in Proceedings of the 8th Symposium (International) on Detonation, ONR Report No. ACR-184 (1989) p187.

7. Sheffield, S.A., Engelke, R., and Alcon, R.R., "Insitu Study of the Chemically Driven Flow Fields in Initiating Homogeneous and Heterogeneous Nitromethane Explosives", in Proceedings of the 9th Symposium (International) on Detonation OCNR Report No. $113291-7$ (1989) p39.

8. Bourne, N.K., Meas. Sci. Technol., 14 (2003) p273278.

9. Millett, J.C.F. and Bourne, N.K., Meas. Sci. Technol., 14 (2003) p601-606.

10. Bourne, N.K., and Milne, A.M., J. Appl. Phys. 95 (2004) p2379.

11. Van Thiel, M. (Ed.), "Compendium of Shock Wave Data”, LLNL Report UCRL-50108, p197.

12. Cau, J.F., "Inappropriate Use of Inclined Electromagnetic Velocity Gauges in Explosives", Proceedings of the 10th International Detonation Symposium, ONR Report No. 33395-12 (1995) p224. 